\title{
Intraorbital Penetrating and Retained Foreign Bodies - A Neurosurgical Case Series
}

\author{
Bianca SZABO ${ }^{1,2^{*}}$, Raluca PASCALAU $3^{3^{*}}$, Dana BARTOS $2,4^{*}$, Adrian BARTOS ${ }^{4^{*}}$, loan SZABO $5^{*}$ \\ ${ }^{1}$ Clinical Emergency County Hospital Cluj, Department of Ophthalmology, Cluj-Napoca, Romania \\ 2"Iuliu Hațieganu" University of Medicine and Pharmacy, Department of Anatomy and Embriology, Cluj-Napoca, Romania \\ 3"Iuliu Hatieganu" University of Medicine and Pharmacy Cluj-Napoca, Faculty of Medicine, Cluj-Napoca, Romania \\ ${ }^{4}$ Regional Institute of Gastroenterology and Hepatology, Department of Surgery, Cluj-Napoca, Romania \\ ${ }^{5}$ Cluj County Emergency Hospital, Department of Neurosurgery, Cluj-Napoca, Romania
}

*The authors have contributed equally to conceiving, writing and reviewing the manuscript so that they should all be considered main authors.

This study has been presented at the Al Xix-Lea Congres Al Societății Române De Anatomie between 17 and 19 May 2018, at Cluj-Napoca, Romania.

Corresponding author: Raluca PASCALAU raluca.pascalau@yahoo.ro

\section{ABSTRACT}

AIM: To present the particularities of the intraorbital foreign bodies from a neurosurgical perspective by summarizing the findings of a case series.

MATERIAL and METHODS: A retrospective study was conducted including a consecutive series of 30 patients with intraorbital foreign bodies treated between 1999 and 2017. Statistical analysis was performed in order to characterize the factors that influence the location of the foreign bodies and the clinical signs.

RESULTS: The orbital trauma occurred mostly in working accidents. Multiple intraorbital foreign bodies were found in $23.3 \%$ of the patients. Metallic foreign bodies were seen in $66.6 \%$, and $30 \%$ had wooden foreign bodies. Nonmetallic foreign bodies were significantly associated with displacement of the eyeball, palpebral oedema and upper lid ptosis. Posterior orbit location was associated with displacement of the eyeball and conjunctival hemorrhage. Intraconal location was associated with mydriasis and conjunctival hemorrhage. The posterior orbit was occupied by foreign bodies in $63 \%$ of the patients. The foreign bodies were in the extraconal compartment in $55.55 \%$ of the cases. Small foreign bodies tend to be retained in the anterior orbit while large ones tend to be retained in the posterior orbit.

CONCLUSION: The diagnosis and management of intraorbital foreign bodies must be tailored according to their type and location and to the clinical aspect of the patient.

KEYWORDS: Intraorbital foreign bodies, Orbital trauma, Neurosurgery

\section{INTRODUCTION}

A $\mathrm{n}$ intraorbital foreign body (IOFB) is defined as an object located inside the bony orbital walls, posterior to the orbital septum but outside the ocular globe $(10,38)$. Its position can be extraconal or intraconal (37), and it can be classified as metallic or nonmetallic; in turn, nonmetallic IOFBs can be organic or inorganic (38). IOFBs typically occur in males, children, and young adults with a history of work accidents or gunshot injuries involving high-velocity projection $(2,10,22,29)$.

Though some cases appear to be routine, special attention must be given to remnant foreign bodies because underestimating trauma can have dangerous or potentially life-threatening consequences (5), including vision loss due to lesions of the
Bianca SZABO (D) : 0000-0002-2605-8513

Raluca PASCALAU (D) : 0000-0002-7059-6896
Dana BARTOS : 0000-0002-7523-4747

Adrian BARTOS (D) : 0000-0003-3177-9232
Ioan SZABO (D) : 0000-0003-3127-4031 
eye ball, optic nerve, or their vasculature (8); lesions of the abducens or oculomotor nerves (26); orbital inflammation (2); orbital fistula (38); orbital compartment syndrome (18); or even pneumocephalus or meningitis (13). As clinical signs at the anterior surface of the orbit can be sparse (29), nonspecific such as in orbital cellulitis (17), or present late after the orbital trauma when the entry wound is healed (22), the presence of an intraorbital foreign body should be suspected in every case of orbital trauma, and this diagnosis should be confirmed or excluded on imaging.

As a general recommendation, empirical broad-spectrum antibiotics should be administered in each case, and antifungal treatment should be used in cases of wooden foreign bodies $(27,36)$. Removal of the intraorbital foreign body can be very difficult due to the anatomical complexity of the orbit, which is a small space with a high density of functional elements (34).

The particularities of the orbital region, being at the limit between the neurocranium and the viscerocranium and the context of orbital trauma raise the need for a complex multidisciplinary management including neurosurgery, ophthalmology, otolaryngology, maxillofacial surgery and plastic surgery $(12,23,26,30)$.

Each specialty involved in the management of IOFBs has its own perspective regarding this pathology that is influenced by the signs and symptoms of the patient. Moreover, the literature regarding this pathology is relatively limited, particularly concerning foreign bodies located in the posterior orbit (27). The majority of published articles about IOFBs are case reports or small case series. We identified only 5 case series with at least 25 patients $(6,15,16,30,35)$, the largest one reporting 53 cases (6). Of these 5 studies, 4 were ophthalmological case series and 1 was a plastic surgery case series (35). The results of these studies cannot be directly extrapolated to the neurosurgical cases due to the different histories, clinical features, and treatment options. The largest neurosurgical series consisted of 4 cases (30), which can be explained by the fact that this pathology is rare in a neurosurgical clinic (31). The aim of the present paper was to report the characteristics of a larger neurosurgical case series in terms of diagnosis and treatment of different categories of foreign bodies, according to their material and location in the orbit, as a reference for neurosurgeons who encounter this pathology.

\section{MATERIAL and METHODS}

We conducted a single-center, single-surgeon, retrospective study by including a consecutive series of 30 patients with IOFBs who were admitted to our neurosurgery clinic between 1999 and 2017. Inclusion criteria were the presence of a retained foreign body in the orbit, documented on imaging and admission to the neurosurgical department. The presence of associated cranial or cerebral lesions and insufficient clinical data were the exclusion criteria. The following data was documented for each patient: gender, age, cause of the orbital trauma, material, size and location of the foreign body, clinical features, imaging findings, time from orbital trauma to complete removal, surgical approach, and postoperative status. The location was classified according to the compartments of the orbit. We calculated the frequency of the findings in the case series. In addition, we performed statistical analysis using the Chi-square test to characterize the factors influencing the location of the foreign bodies in the different orbital compartments as well as the prevalence of clinical signs. The significance threshold was set at 0.05.

Written informed consent was obtained from the patients. The present study was performed in accordance with the principles of the Declaration of Helsinki. The study protocol was approved by the Ethics Committee of our university.

\section{RESULTS}

\section{General Data}

Between 1999 and 2017, 30 patients with IOFBs were treated by the same neurosurgeon in the Neurosurgery Department of the Cluj County Emergency Hospital, Cluj-Napoca, representing $18.75 \%$ of the 160 cases of orbital trauma and $2.17 \%$ of the 1380 total cases of orbital pathology during this period. Patients in this case series were predominantly male (29 males to 1 female) with an average age of 35.7 years and an age range of 4-63 years. IOFBs were typically encountered in young and middle-aged adults and small children (Figure 1).

In most of the cases, orbital trauma occurred in work accidents (50\% of the cases), especially wood chopping, followed by accidental falls (30\%), and gunshots (16.66\%). The time period from the orbital trauma to getting admitted to the neurosurgical clinic ranged from 6 hours to 7 years.

Of patients, $76.6 \%$ presented with single foreign bodies, whereas $23.3 \%$ had multiple IOFBs, with all of them located unilaterally. As a result, the 30 patients in the present study had a total of 42 IOFBs with a maximum diameter ranging from $0.1 \mathrm{~cm}$ to $6 \mathrm{~cm}$, with a mean value of $1.3 \mathrm{~cm}$. The shapes of the foreign bodies were diverse (Figure 2A-I). Twenty one metallic foreign bodies, out of which one was mixt-metal and enamel, were encountered in $66.6 \%$ of patients. Twenty wooden foreign bodies, out of which one was mixt-a pencil, appeared in $30 \%$ of patients, and one foreign body was made of nonmetallic material other than wood (Figure 2A-I).

\section{Clinical Features}

The most frequently manifesting clinical symptoms of traumatic optic neuropathy were orbital pain, diplopia, and decreased vision or visual field defect. The pathognomonic sign was the presence of an open or closed entry wound. In our cases, the entry wound was located primarily on the eyelids or in the conjunctival fornix (Figure 3). The foreign bodies retained in the anterior orbit entered through the conjunctival fornix or the eyelid, where as those retained in the posterior orbit mainly penetrated the eyelid or the sclera, followed by the fornix or the cornea. The distribution is similar if we consider the extraconal vs. intraconal space distribution. According to the material, the metallic foreign bodies leaved entry wounds the most frequently in the conjunctival fornix, while all the nonmetallic foreign bodies penetrated the eyelids. 


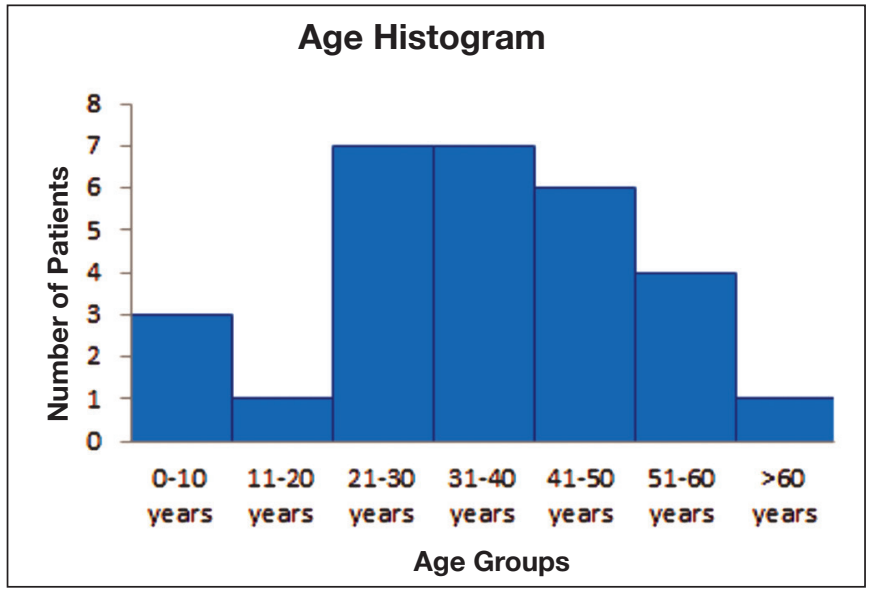

Figure 1: Age distribution of the patients with intraorbital foreign bodies.
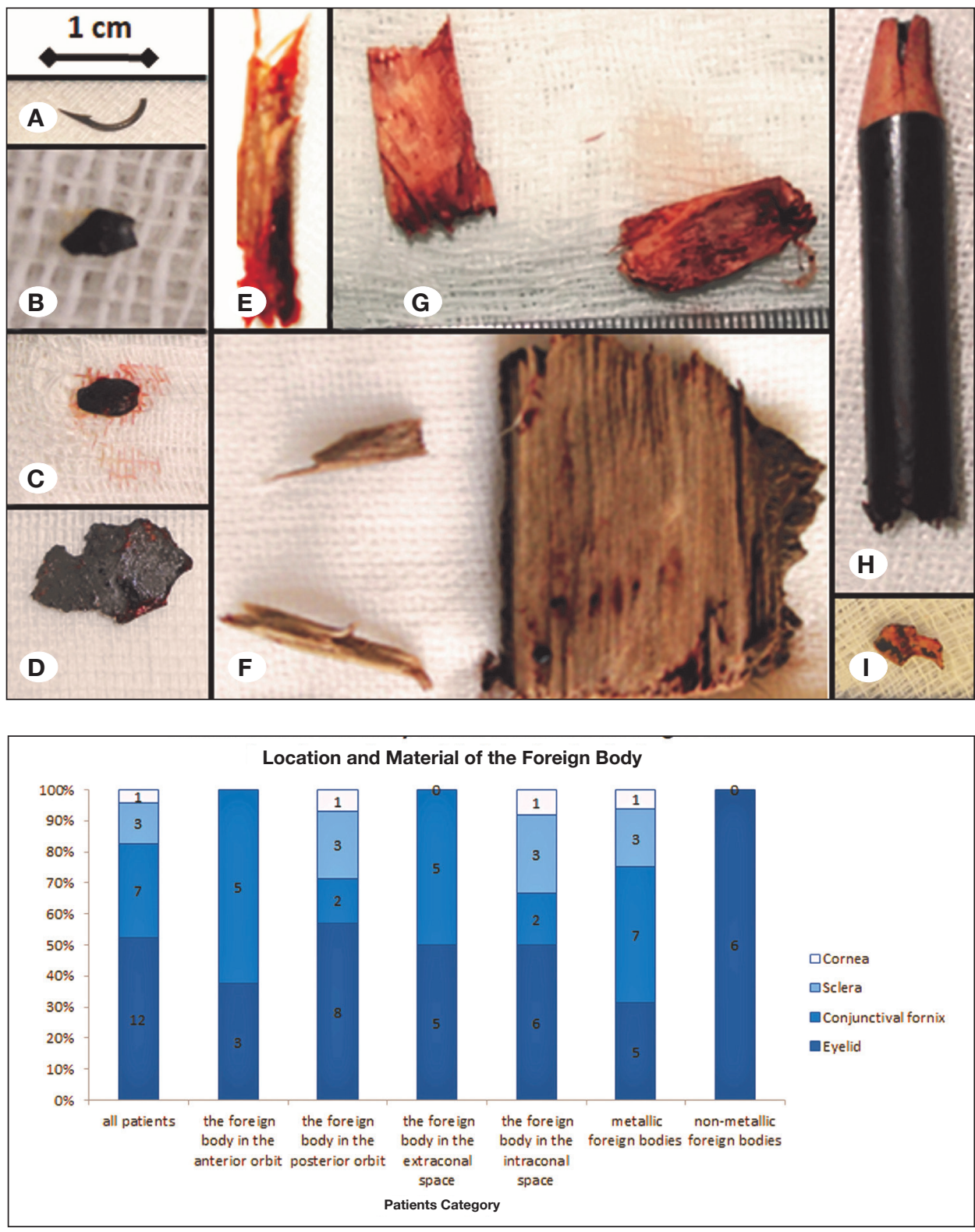

The most often encountered associated clinical signs were: conjunctival congestion, displacement of the eyeball, upper lid ptosis, mydriasis, palpebral oedema, conjunctival hemorrhage, palpebral ecchymosis, limited eye movements, exophthalmos and chemosis (Figures 4A-E, 5). Other less common signs were also observed such as uveitis $(15.38 \%$ of the patients), retinal detachment (15.38\%), orbital infection (15.38\%), hemophthalmos (11.53\%), traumatic cataract $(7.7 \%$ of the patients), epiphora (37.7\%) or blepharospasmus (3.5\%) (Figures 4A-E, 5).

The prevalence of clinical signs varies in different categories of patients according to the location of the foreign body material (Figure 6A, B). Statistical analysis using the Chi-square test revealed significant associations between displacement of the eyeball and the posterior orbit location (Chi-square test: 6.1348 , and $p=0.013255)$, mydriasis and intraconal location

Figure 2: Examples of foreign bodies (FBs). A) Metallic FB, fishing hook. B) Metallic FB, fragment of a hammer head. C) Metallic FB. D) Metallic FB. E) Wooden FB. F) Multiple wooden FBs. G) Multiple wooden FBs. H) Mixt wooden FB, pencil. I) Mixt FB, metal and enamel.

Figure 3: Distribution of the entry wound location according to the location and material of the foreign body. 
(Chi-square test: 3.8845 , and $p=0.048734$ ), conjunctival hemorrhage and posterior orbit location (Chi-square test: 4.7902, and $p=0.028622$ ), and conjunctival hemorrhage and intraconal location (Chi-square test: 3.8845, and $\mathrm{p}=$ $0.048734)$. Statistically significant associations were found between displacement of the eyeball and nonmetallic foreign bodies (Chi-square test: 10.4 , and $p=0.00126)$, upper lid ptosis and nonmetallic foreign bodies (Chi-square test: 5.85, and $\mathrm{p}=0.015577$ ), and palpebral edema and nonmetallic foreign bodies (Chi-square test: 4.6265 , and $p=0.031482$ ).

\section{Diagnosis in Neuroimaging}

The imaging modality was chosen according to the presumed material of the foreign body (Figure 7A-L). The most frequently utilized imaging tool, regardless of the foreign body material, was computed tomography (CT) ( $88 \%$ of the cases). Overall,

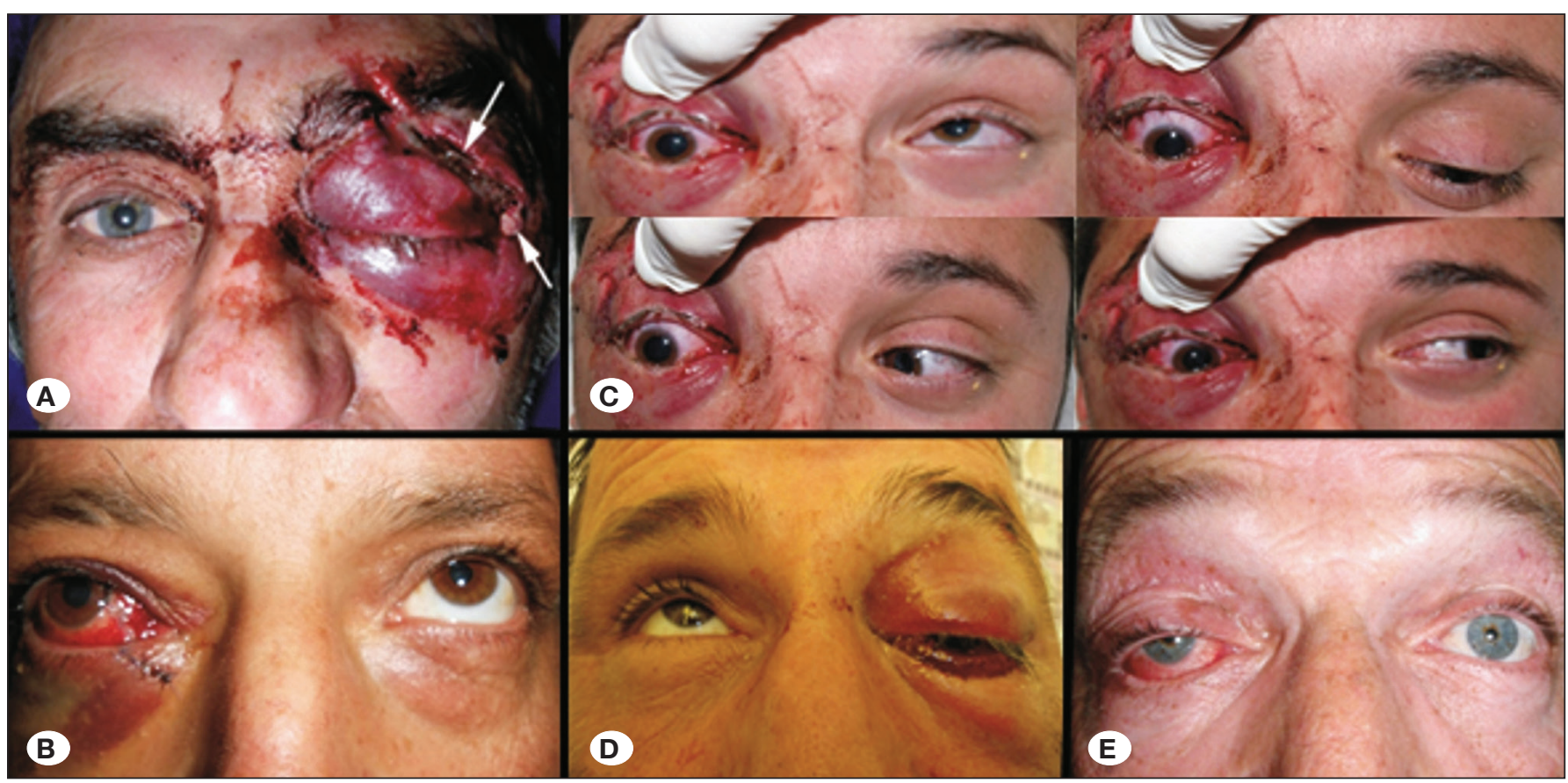

Figure 4: Clinical aspects. A) Oedema, ecchymosis and ptosis. B) Ecchymosis, conjunctival hemorrhage and mydriasis. C) Oedema, ptosis, limited superior and lateral movements, chemosis, mydriasis and epiphora. D) Oedema, ecchymosis, ptosis, exophthalmos limited superior and medial movements, conjunctival hemorrhage, chemosis and mydriasis. E) Oedema, ptosis, ectropion, exophthalmos and chemosis.

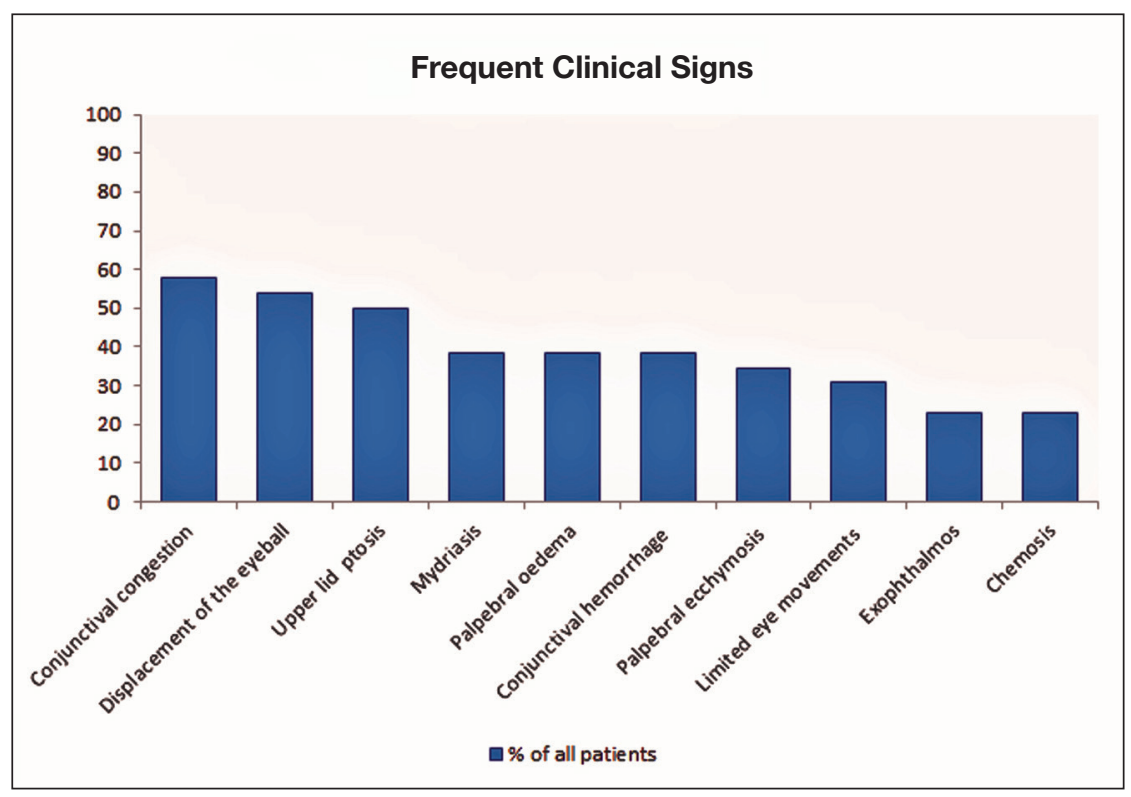

Figure 5: Frequent clinical signs in patients with IOFBs (the percentual prevalence of the clinical signs in decreasing order). 
$87.5 \%$ of the foreign bodies were hyperdense, $8.3 \%$ hypodense, and $4.15 \%$ presented with mixed heterogeneous density. All of the metallic foreign bodies appeared hyperdense with artifacts on the brain window and with their proper dimensions on the bone window. Wooden foreign bodies appeared hypodensesimilar to air-in the acute phase and heterogeneous isodense in the intraorbital muscles or even hyperdense in the subacute or chronic phase. The brain window was also useful for detecting intraorbital emphysema $(25.9 \%)$ or lesions of the orbital structures, such as the ocular globe $(29.6 \%)$ or the optic nerve (18.5\%). Three dimensional (3D)-reconstruction CT scan was specifically helpful in determining the position of the orbital foreign bodies and in surgical planning. Orbital radiography using Comberg loupe was used in $22 \%$ of the patients, all of them with metallic foreign bodies appearing to be radiopaque. Evaluation of each case was concluded with $\mathrm{CT}$ imaging. Magnetic resonance imaging (MRI) was used in
$18 \%$ of the patients, all of whom presented with nonmetallic foreign bodies. Wooden foreign bodies may have increased signal intensity related to water content and changes in time, as the foreign body absorbs blood or other biological fluids. In the study of the dimensions and location of the foreign bodies,T1-weighted sequences were more useful than T2weighted sequences; 3D-MRI reconstruction was also performed. In addition, orbito-ocular ultrasonography was performed in $11 \%$ of the patients, especially in cases with intraconal foreign bodies in close proximity to the optic nerve. The central retinal artery was visualized with the Doppler mode (Figure 7A-L).

\section{Foreign Body Location}

The IOFBs were found in the right orbit in $60 \%$ of the patients. In $37 \%$ of the cases the foreign bodies were in the anterior orbit. The posterior orbit was occupied by foreign bodies in

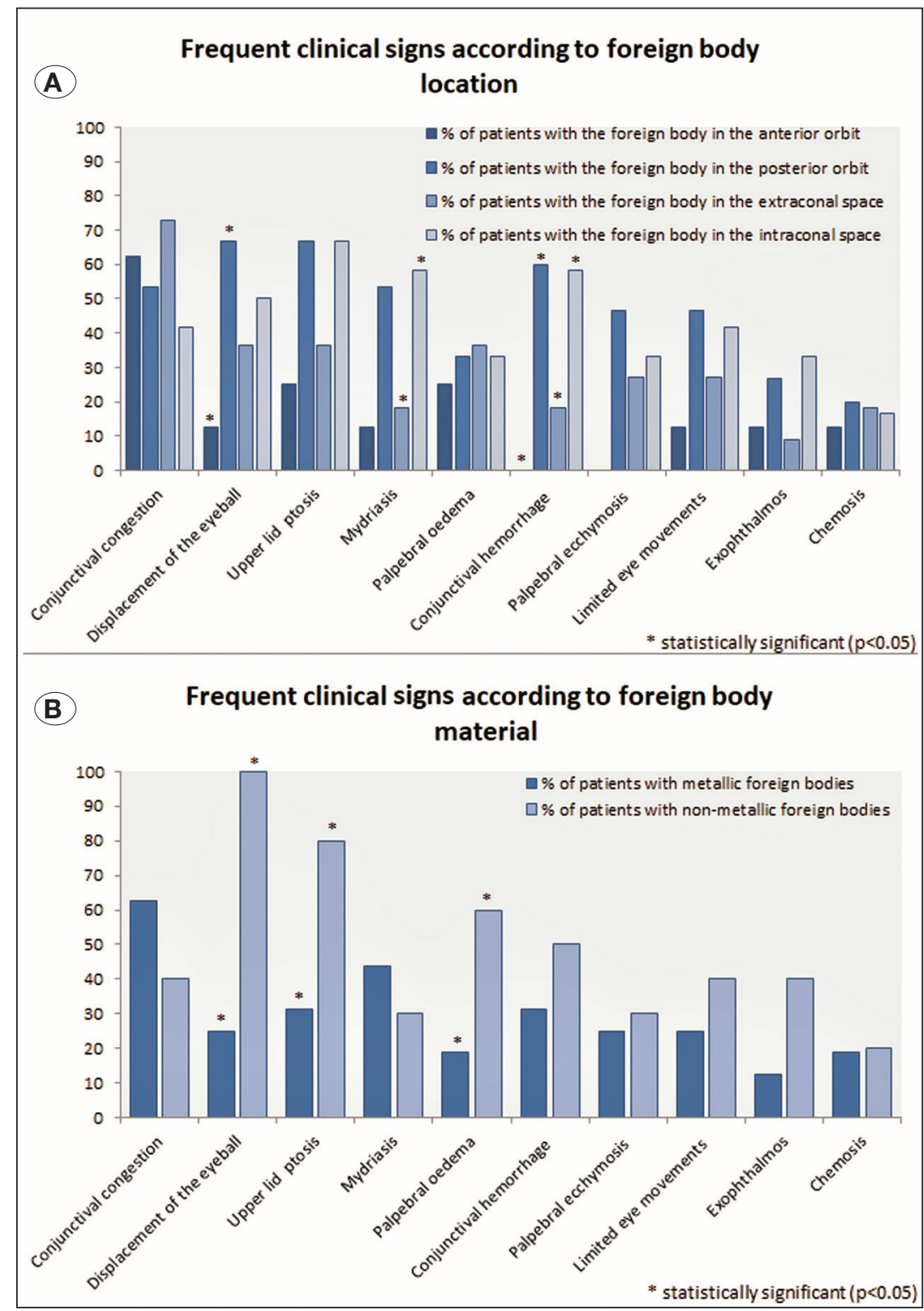

Figure 6: A) Association between the foreign body location and the percentual prevalence of the clinical signs. B) Association between the foreign body material and the percentual prevalence of the clinical signs. 
$48 \%$ of the patients, $11 \%$ having them in the medium orbit and $37 \%$ in the retrobulbar compartment. Another $14.8 \%$ of the patients had large foreign bodies that occupied the entire sagittal length of the orbit.

As seen in Figure 8, the distribution of foreign bodies in the different orbital spaces is influenced by their material and size. While metallic foreign bodies were retained mostly in the posterior orbit (57.14\%) and in the extraconal space (62\%), nonmetallic foreign bodies also occupied the posterior orbit $(72.72 \%)$, but they were distributed evenly between the extraconal and intraconal spaces. Foreign bodies with the maximum diameter smaller than the average value tended to be retained in the anterior orbit $(55 \%)$ and in the extraconal space $(68.42 \%)$ as opposed to those with the maximum diameter larger than the average value, which were more frequently located in the posterior orbit $(92.3 \%$ of the large foreign bodies) and in the intraconal space $(57.14 \%$ of the small foreign bodies); these were the only statistically significant associations on the Chi-square test (Chi-square test: 7.6199 , and $\mathrm{p}=0.005773$ ).

\section{Surgical Treatment}

Out of the 30 patients included in the case series, $93.33 \%$ were operated under general anesthesia, with oro-tracheal intubation. Prior to the surgery the patients received broad spectrum antibiotics, including the anaerobic flora. In $76.66 \%$ of the cases the foreign bodies could be extracted in single interventions, while $16.66 \%$ of the patients needed 2 or 3 interventions out of which at least one, not necessarily the first one, was performed in the neurosurgical clinic and the others in ophthalmological or maxilo-facial surgery clinics. The surgical approach was chosen according to the location of the foreign body (Figure 9A-F). If possible, the foreign body was extracted by use of the entry route, by opening and enlarging the scar. $11.11 \%$ of the cases were operated with intraoperative ultrasound guidance. The most used approach was the anterior transpalpebral approach (59.25\%) followed by the anterior transconjunctival approach and the lateral approach with the same frequency $(18.51 \%)$. The superior transfrontal approach was used only in one patient.

Location of the foreign bodies influenced the type of surgical approach used (Figure 10). The anterior transpalpebral approach was used most commonly for those foreign bodies located in the anterior orbit. For this approach, a horizontal skin incision was made parallel to the eyelid margin, preserving the orbicularis muscle. The orbital septum was incised and the lateral or medial canthal ligaments were disinserted if required, based on the location of the foreign body. The extraconal space was entered and the extraconal foreign body was extracted. Intraconal foreign bodies were extracted by accessing the intraconal space and retracting the extraocular muscles under ultrasound guidance, while identifying and preserving

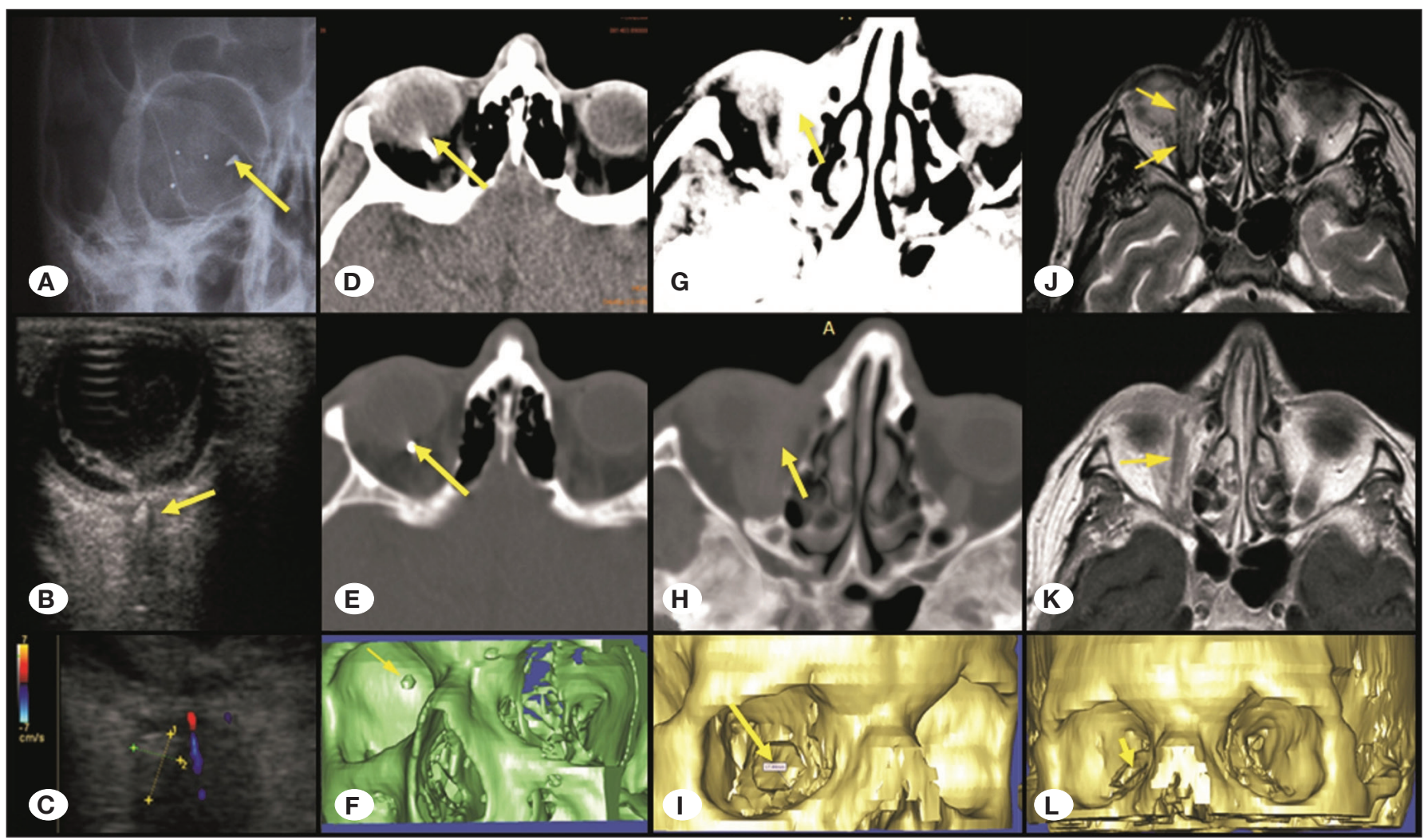

Figure 7: Imaging studies: A-F intraconal metallic FB and G-L extraconal wooden FB. A) X Ray. B) B-mode ultrasonography. C) ColorDoppler ultrasonography. D) Axial CT scan, brain window. E) Axial CT scan, bone window. F) 3D-CT. G) Axial CT scan, brain window. H) Axial CT scan, bone window. I) 3D-CT. J) MRI-T1 sequence. K) MRI-T2 sequence. L) 3D-CT scan. 
the anatomical structures (branches of the ophthalmic artery, ophthalmic veins, and branches of the cranial nerves and the optic nerve). The wound was closed by suturing the orbital septum, reinserting the palpebral ligaments, and suturing the skin in separate knots; it was dressed with a topical antibiotic ointment and a cotton patch. For retrobulbar foreign bodies, the percentage of cases undergoing anterior approaches was the lowest while the lateral approach was used in $45 \%$ of the cases. The lateral approach involved a Krönlein hockeystick incision, disinsertion of the lateral canthal ligament from the Whitnall tubercle, removal of the frontozygomatic arch and the lateral orbital wall, sectioning the periorbita in an "H-shaped" manner, and entering the extraconal space. The intraconal space was accessed from above or below the lateral rectus muscle according to the location of the foreign bodies. The foreign bodies were extracted under ultrasound guidance, with care taken to preserve the ciliary ganglion and the other anatomical structures. The periorbita was closed using running sutures, the lateral canthal ligament was reinserted, the bone was placed in its original position and fixed with threads, and the wound was closed. Foreign bodies occupying the entire orbit were extracted using an anterior transpalpebral approach, except for 1 case that required a second surgery with a superior transfrontal approach for

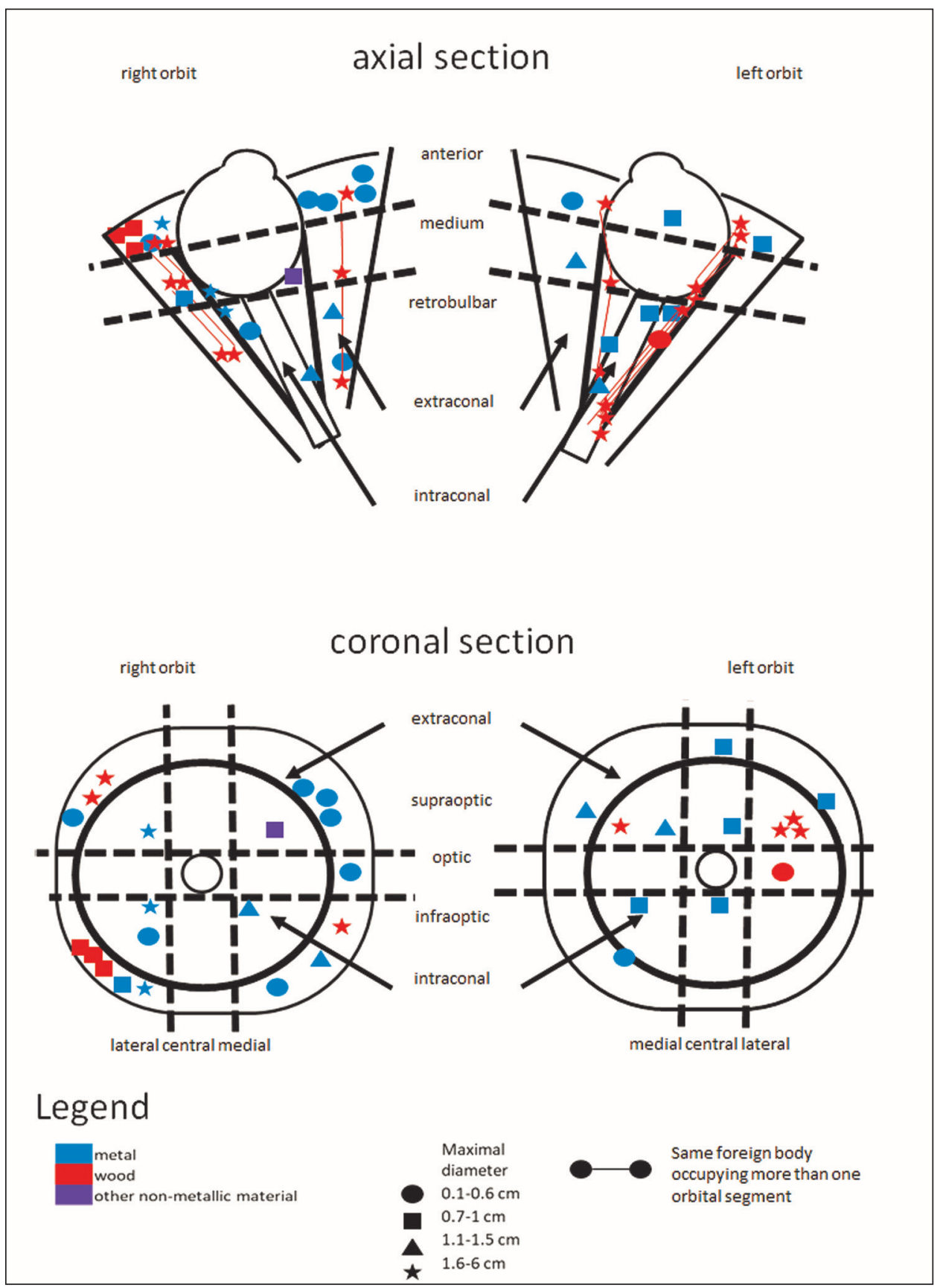

Figure 8. Schematic illustration of the location of the foreign bodies in the compartments of the orbit. 


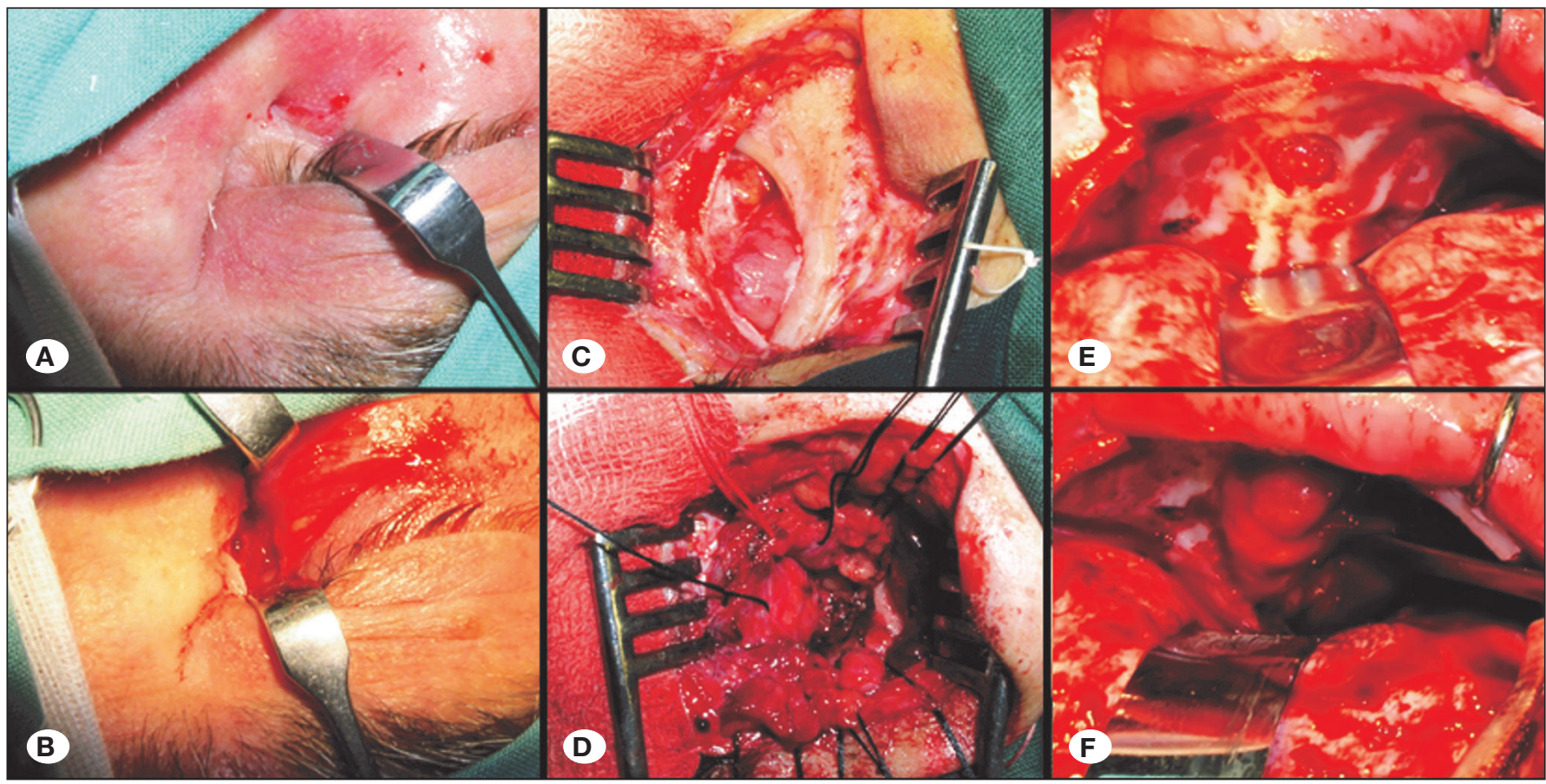

Figure 9: Surgical approaches. A, B) Anterior transpalpebral approach using the foreign body entry wound on the eye lid. C, D) Lateral approach with lateral osteotomy. E, F) Superior transfrontal approach.

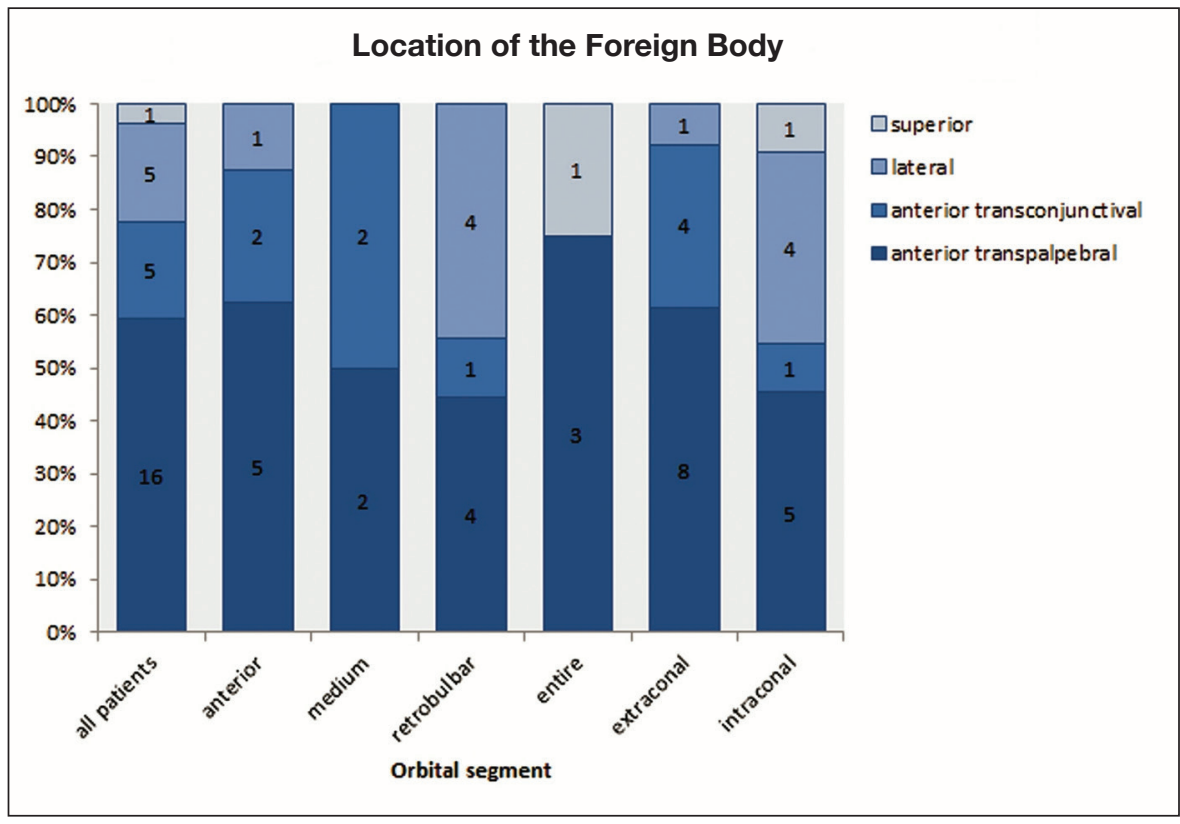

Figure 10: The choice of the surgical approach according to the location of the foreign body.

a fragment entrapped in the optic canal (Figure 9E, F). The superior transfrontal approach required a unilateral frontal craniotomy, dura opening, frontal lobe retraction, and removal of the optic canal roof and the posterior part of the superior orbital wall. The periorbita was incised with special care to preserve the frontal nerve, and the intraconal space was entered by medially or laterally retracting the superior rectus/ levatorpalpebrae complex. After extracting the foreign body, the periorbita was closed with running sutures, and titanium mesh was used to reconstruct the superior orbital wall. The dura was sutured, the bone flap was repositioned and fixed with threads, and the scalp was closed in anatomical planes.

Hemostasis was achieved by compression or bipolar coagulation if necessary. Periorbital/dural lacerations were repaired by running sutures, if possible, or using free periosteal flaps. Associated orbital wall fractures were treated by removing the splinters and drilling the bone edges. 
In the majority of the cases, the foreign bodies were removed with good postoperative results. Vision, pupils, and ocular motility were checked every hour on the day of surgery. The most common immediate postoperative status included upper lid ptosis in $27 \%$ of the patients, palpebral edema in $18 \%$, conjunctival congestion in $18 \%$, and chemosis in $8.33 \%$. Antibiotic therapy was continued, with adjustments made according to the results of the microbiological analysis of the extracted material together with analgesics and antiinflammatory medication. The patients were followed up at 1 month after the surgery and annually there after.

\section{DISCUSSION}

IOFBs represent an uncommon pathology in neurosurgical practice. Whereas they account for $3 \%$ of the consultations in emergency departments in the U.S. (10), in our department they occurred in only $2.17 \%$ of the orbital pathology, which has a variable percentage from one neurosurgeon to another. Among the orbital trauma cases, they occupied nearly one fifth compared to one sixth of those reported in the literature $(8,28)$. The male dominance, the young age and the working accidents as leading cause of trauma were consistent with other series reported $(22,29)$. Similar to ophthalmological (14), and maxillofacial series (29), the most of the foreign bodies were metallic.

When an intraorbital foreign body is suspected, the first step is to look for the entry wound. According to our findings, the eyelids should be searched first, followed by the conjunctival fornix, and if the foreign body is located in the posterior orbit, the entry wound could be on the sclera or cornea, and a double perforation of the ocular globe may be present (18). Regarding the clinical signs associated to IOFBs, the most frequent are optic nerve lesion, limitation of ocular motility, exophthalmia and infection (21). Our study is the first to have identified statistically significant factors influencing the clinical signs of a foreign body. Foreign bodies of the anterior orbit were associated with local inflammatory signs, while those retained in the posterior orbit correlated not only with inflammatory signs but also hemorrhage and functional deficits, such as mydriasis and displacement of the globe. The same tendency was observed in extraconal compared to intraconal foreign bodies. While metallic foreign bodies except for copper are well tolerated if the globe is not injured (18), the organic foreign bodies, especially wooden ones, are linked to a high risk for bacterial and fungal infections, inflammatory granuloma formation, and calcification $(21,22)$. In our series, nonmetallic foreign bodies, mostly wooden, were more aggressive than the metallic foreign bodies, leading to a higher prevalence of clinical signs such as palpebral edema, upper lid ptosis, and displacement of the eyeball. They also have a porous structure that enables the growth of microorganisms (8). Infection resulting from retained intra-orbital wooden foreign bodies may lead to complications such as panophthalmitis, abscess, and chronic fistula (35). Orbital infection of various severity grades was relatively frequent in our series, $15.38 \%$ of the cases.
Orbital ultrasonography was used in the present study, especially for intraconal foreign bodies, but this technique must not be performed in cases of ruptured globe (10). The disadvantage of orbital ultrasonography is that orbital emphysema, which is often present in IOFBs, impairs the examination. In addition, the quality of the evaluation also depends on the experience of the examiner (3). CT is the most commonly recommended imaging method, with the highest sensitivity for better localization of the foreign body (25), especially metallic foreign bodies. In the present study, all metallic foreign bodies were hyperdense as well as easily detected, localized, and measured on CT. Nonmetallic foreign bodies can also be detected on CT but with some difficulty. In our series, wooden foreign bodies had variable density. They can be mistaken for intraorbital air in the acute phase, intraorbital fat in the subacute phase, and the extraocular muscles in the chronic stage (17). In such cases, indirect signs, such as structure displacements, must be considered. MRI is highly recommended after excluding the possibility of metallic foreign bodies (28). Wood may appear hypointense or hyperintenseon MRI depending on the water content (24). The advantage of MRI over CT is that surrounding inflammation or hematoma can be better delineated from the foreign body, especially on T2 sequences where it appears hyperintense (24). 3D reconstructions based on CT or MRI are particularly useful for foreign body localization and surgical planning $(5,11)$.

This neurosurgical series is unique regarding the high prevalence of cases with foreign bodies located in the posterior orbit, namely $48 \%$, as opposed to $9 \%$ in a plastic surgery series (35), and $12.5 \%$ in a maxillofacial series (29). Metallic foreign bodies reportedly tend to lodge in the posterior orbit due to high momentum, and nonmetallic foreign bodies with a lesser momentum are prone to stop in the anterior orbit (28). However, the Chi-square test in our series found no significant association between foreign body material and orbital location. The only significant association was found between small foreign bodies and anterior orbit location, as opposed to large foreign bodies associated with posterior orbit location.

The therapeutic strategy is influenced by the material and location of the foreign bodies and by the clinical features of the patient, especially the presence of infection $(20,29)$. The high density of the vital structures (33), orbital fat, and connective tissue, which hinder visualization, increase the risk for postoperative complications (7), such as orbital hemorrhage, optic nerve damage, or orbital compartment syndrome (22). Surgical removal of foreign bodies from the posterior orbit has a higher risk for motility disturbance or optic neuropathy (19). Wooden foreign bodies are particularly difficult to remove due to their soft structure, making them prone to fracture during extraction with the risk of remnant fragments and consequent infection (4). Indications for surgical removal of foreign bodies include sharp foreign body, organic material, foreign body toxicity, mechanical restrictions of ocular motility, neurological deficits, compression of the intra-orbital structures, exophthalmia, development of infection, orbital fistula formation, and pain $(1,10,12,24,27)$. In contrast, surgical removal is not required for blunt inorganic, asymptomatic, or deeply located foreign bodies (10). The approach should 
provide good access, good hemostasis, safety for the intraorbital structures (4), and the entry wound should be used when possible $(5,12,22)$. Maxillofacial and plastic surgeons mostly use anterior approaches $(29,35)$, and ophthalmologists use anterior and lateral approaches (14). The endoscopic transnasal approach is used only by ear, nose, and throat surgeons (23). Neurosurgeons are familiar with the anterior and lateral approaches (34), and are the only specialists that perform the superior transfrontal approach, which provides good access to the orbital apex and less traction on the ocular globe, which is very important in cases of perforated globe (9). Foreign bodies located in the anterior orbit are usually removed using an anterior approach (22). Anterior approaches can also be used in foreign bodies retained in the posterior orbit if they are accessible, but deeply situated foreign bodies should be extracted using a lateral approach, which provides good access to the lateral orbit and the intraconal space (32) and is less aggressive compared to the superior transfrontal approach (34). Intraoperative ultrasound guidance is useful, especially when the foreign body is very close to the optic nerve.

\section{CONCLUSION}

Patients with IOFBs who are admitted to a neurosurgical clinic should be thoroughly examined, including for the entry wound on the eyelids, conjunctival fornix, or the ocular surface. Nonmetallic objects and foreign bodies located in the posterior orbit or the intraconal space have numerous clinical manifestations. CT imaging is recommended for localizing the foreign bodies, evaluating the intra-orbital structures, and surgical planning. In addition, MRI is useful once a metallic object is excluded. IOFB management must be tailored according to their type and location as well as to the clinical characteristics of the patient. Organic foreign bodies must be completely removed and broad-spectrum antibiotics administered that cover the anaerobic flora. Metallic foreign bodies are better tolerated; hence indications for removal must be individually assessed.

\section{- REFERENCES}

1. Al-Amry M, Al-Saikhan FI, Al-Dahmash S: External ophthalmomyiasis: A case report. Saudi J Ophthalmol 28: 322-324,2014

2. Arora V, Kim UR, Shetty S, Shah AD: Atypical presentation of an unusual foreign body. Indian J Ophthalmol 58:338339,2010

3. Bailey W, Robinson L: Screening for intra-orbital metallic foreign bodies prior to MRI: Review of the evidence. Radiography 13:72-80,2007

4. Bater MC, Scott R, Flood TR: Use of an inferior orbitotomy for safe removal of a wooden foreign body penetrating the orbit. Brit J Oral Max Surg 45: 664-666, 2007

5. Blackhall KK, Laraway DC: Penetrating retro-orbital foreign body - large glass shards: A maxillofacial surgery case report. SAGE Open Medical Case Reports 4:1-5,2016

6. Callahan AB, Yoon MK: Intraorbital foreign bodies: Retrospective chart review and review of literature. Int Ophthalmol Clin 53:157-165,2013
7. Cho RI, Kahana A, Patel B, Sivak-Callcott J, Buerger DE, Durairaj VD, Vidor I, Mawn LA: Intraoperative fluoroscopyguided removal of orbital foreign bodies. Ophthal Plast Reconstr Surg 25:215-218,2009

8. Cho WK, KO AC, Eatamadi H, Al-Ali A, Abboud JP, Kikkawa DO, Korn BS: Orbital and orbitocranial trauma from pencil fragments: Role of timely diagnosis and management. Am J Ophthalmol 180: 46-54, 2017

9. Chung IY, Seo SW, Han YS, Kim E, Jung JM: Penetrating retrobulbar orbital foreign body: A transcranial approach. Yonsei Med J 48:328-330, 2007

10. Czyz CZ, Petrie TP, JD Harder, Cahill KV, Foster JA: Intraorbital foreign body projectile as a consideration for unilateral pupillary defect. Int J Emerg Med 5:14,2012

11. De Morais HHA, Barbalho DCM, de Souza Dias TG, Grempel RG, de Holanda Vasconcellos RJ: Temporal approach to removal of a large orbital foreign body. Cranio Maxillo Facial Trauma and Reconstruction 8: 234-238, 2015

12. Dell'Aversana G, Marenzi G, Piombino P, Testa D, De Riu G, Abbate V, Califano L, Sammartino G: Violence-related periorbital trauma with a retained foreign body: A case report. J Med Case Rep 20:10-16,2016

13. Detorakis ET, Symvoulakis EK, Drakonaki E, Halkia E, Tsilimbaris MK: Unexpected finding in ocular surface trauma: A large intraorbital foreign body (Bullet). Acta Med (Hradec Králové) 55:100-103,2012

14. Dolar Bilge A, Yılmaz H, Yazıcı B, Naqadan F: Intraorbital foreign bodies: Clinical features and outcomes of surgical removal. Ulus Travma Acil Cerrahi Derg 22:432-436,2016

15. Finkelstein M, Legmann A, Rubin PA: Projectile metallic foreign bodies in the orbit: A retrospective study of epidemiologic factors, management, and outcomes. Ophthalmology 104: 96-103,1997

16. Fulcher TP, McNab AA, Sullivan TJ: Clinical features and management of intraorbital foreign bodies. Ophthalmology 109: 494-500,2002

17. Gupta K, Kaushal JB: Giant orbital foreign body mimicking as orbital cellulitis: A rare case report. SEAJCRR 32: 2319-1090, 2014

18. Hada M, Chandra, Gangwe A: An unusual case of protruding retro-orbital metallic foreign body. Indian $\mathrm{J}$ Ophthalmol 64: 604-606,2016

19. Hung KH, Yang CS, Kao SC, Lee FL, Lee SM: Management of double-penetrating ocular injury with retained intraorbital metallic foreign body. J Chin Med Assoc 74: 523-526,2011

20. Jiang $H, X u X, X i a X$ : Surgical method and intraorbital foreign body extraction in 27 cases. Zhong Nan Da XueXueBao Yi Xue Ban 35: 1013-1017,2010

21. Khairuddin O, Suraida AR, Tai LME, Adil H, Liza-Sharmini AT, Mohtar I: Lower lid entropion secondary to missed intraorbital foreign body in a child. Open Journal of Ophthalmology 6: 198-204,2016

22. Li J, Zhou LP, Jin J, Yuan HF: Clinical diagnosis and treatment of intraorbital wooden foreign bodies. Chin J Traumatol 19: $322-325,2016$ 
23. Li W, Wang Y: Long wooden stick penetrating across the retromandibular, nasopharyngeal and contralateral orbital region in a child. Int J Pediatr Otorhi Extra 4:88-91,2009

24. Lin KY, Ngai P, Echegoyen JC, Tao J: Imaging in orbital trauma. Saudi Journal of Ophthalmology 26: 427-432, 2012

25. Lin TC, Liao TC, Yuan WH, Lee FL, Chen SJ: Management and clinical of intraocular foreign bodies with the aid of orbital computed tomography outcomes J chin Med Assoc 77:433436, 2014

26. Markowski J, Dziubdziela W, Gierek T, Witkowska M, MrukwaKominek M, Niedzielska I, Paluch J: Intraorbital foreign bodies -5 own cases and review of literature. Otolaryngologiapolska 66: 295-300, 2012

27. Peralta RJ, Zoumalan C, Lelli GJ Jr: Posterior intraorbital foreign body: Take it or leave it? Open Reconstructive and Cosmetic Surgery 1:1-3,2008

28. Pinto A, Brunese L, Faggian SA, Guarnieri G, Muto M Romano L: Role of computed tomography in the assessment of intraorbital foreign bodies. Semin Ultrasound CT MR 33: 392-395,2012

29. Santos T, Melo AR, Moraes HH, Almeida Júnior $P$, Dourado E: Impacted foreign bodies in orbital region: Review of nine cases. Arq Bras Oftalmol 73:438-442,2010

30. Schreckinger M, Orringer D, Thompson BG, La Marca F, Sagher O: Transorbital penetrating injury: Case series, review of the literature, and proposed management algorithm. J Neurosurg 114: 53-61,2011
31. Sun G, Yagmurlu K, Belykh E, Lei T, Preul MC: Management strategy of a transorbital penetrating pontine injury by a wooden chopstick. World Neurosurg 95:622.e7-622.e15,2016

32. Szabo B, Crişan D, Tompa I, Szabo I: Isolated neurosarcoidosis. Rom J Morphol Embryol 52:1139-1142,2011

33. Szabo BA, Pascalau R, Padurean VA: Morphometric study of the human brainstem and its neurovascular relations. Turk Neurosurg 2017 (Epub ahead of print)

34. Szabo I, Szabo B, Popescu LA, Petrescu M: Orbit evisceration: Retrospective study on a consecutive series of 10 years. Oftalmologia 57: 40-47,2013

35. Taş S, Top H: Intraorbital wooden foreign body: Clinical analysis of 32 cases, a 10-year experience. Ulus Travma Acil Cerrahi Derg 20: 51-55,2014

36. Torres IJ, Burks SS, Levine CG, Bhatia RG, Casiano R, Bullock $\mathrm{R}$ : Wooden foreign body in the skull base: How did we miss it? World Neurosurg 92: 580e5-580e9,2016

37. Turliuc DM, Costan VV, Cucu Al, Costea CF: Intraorbital foreign body. Rev Med Chir Soc Med Nat lasi 119: 179-184,2015

38. Wang X, Xie Q, Wang X, Chen H, Sheng X: Orbital trauma with a large plant foreign body: A case report. Eye Sci 28: 44-47,2013 